\title{
0607 RISK PERCEPTION AND RISKY DRIVING BEHAVIOURS OF ADOLESCENTS AND THEIR PARENTS: NEW ZEALAND DRIVERS STUDY
}

R Brookland*, D Begg, J Langley, S Ameratunga Correspondence: Injury Prevention Research Unit, University of Otago, P.O. Box 913 Dunedin 9054, New Zealand

\subsection{6/ip.2010.029215.607}

Background and Aim A growing body of research indicates that driving behaviours and beliefs of parents can influence their child's driving. However, these studies have been limited by small sample sizes, low recruitment rates or have focused on 'children' with several years of driving experience. The main aim of the present study was to examine the association between risk perception and driving behaviours of parents and their adolescents at the critical time when the young driver transitions into driving solo and crash risk is at its highest.

Hypothesis The risk perceptions and driving behaviours of young newly licensed drivers will reflect their parents' perceptions and behaviours.

Methods This study was part of the New Zealand Drivers Study (NZDS), an ongoing prospective cohort study of 3992 newly licensed car drivers. As part of larger telephone administered interviews, after completing the supervised driving stage of the graduated licensing system, 1200 young drivers (aged 15-17 years) and their parents independently reported their perception of risk regarding unsafe driving practices and their risky driving behaviours.

Results and Conclusion Speeding is a major contributor to injury crashes. Preliminary results indicated significant positive correlations between parent and adolescent risk perception and driving behaviours with regards to exceeding the posted speed limits and driving fast for the thrill of it. These and other findings relating to risky driving will be presented, providing new insights into the relationships between risk perception and risky driving behaviours of young drivers and their parents. 